\title{
The Bethe ansatz as a matrix product ansatz
}

\author{
Francisco C. Alcaraz and Matheus J. Lazo \\ Universidade de São Paulo, Instituto de Física de São Carlos, Caixa Postal 369, 13560-590 São Carlos, São Paulo, Brazil
}

(August 22, 2018)

\begin{abstract}
The Bethe ansatz in its several formulations is the common tool for the exact solution of one dimensional quantum Hamiltonians. This ansatz asserts that the several eigenfunctions of the Hamiltonians are given in terms of a sum of permutations of plane waves. We present results that induce us to expect that, alternatively, the eigenfunctions of all the exact integrable quantum chains can also be expressed by a matrix product ansatz. In this ansatz the several components of the eigenfunctions are obtained through the algebraic properties of properly defined matrices. This ansatz allows an unified formulation of several exact integrable Hamiltonians. We show how to formulate this ansatz for a huge family of quantum chains like the anisotropic Heisenberg model, Fateev-Zamolodchikov model, Izergin-Korepin model, Sutherland model, $t-J$ model, Hubbard model, etc.
\end{abstract}

Since the pioneering work of Bethe in 1931 [1] the Bethe ansatz and its generalizations proved to be a quite efficient tool in the description of the eigenvectors of a huge variety of one-dimensional quantum chains and two dimensional transfer matrices (see e. g. [2]- [5] for reviews). On the other hand in the last two decades [6][9] it has been shown that some special quantum chains, although not integrable through the Bethe ansatz have the components of its ground-state wavefunctions given in terms of a product of matrices. In this matrix product ansatz (MPA), apart from a normalization constant, these components are fixed by the algebraic properties of the matrices defining the MPA. In another context a MPA has also been applied quite successfully to the evaluation of the stationary distribution of probabilities of some stochastic models in one dimension [10]. The time fluctuations of these stochastic models are described by the ground state wavefunction of a related spin Hamiltonian. The simplest example is the one-dimensional asymmetric exclusion process [10] whose related spin Hamiltonian is the anisotropic Heisenberg chain, or XXZ chain, with appropriate boundary fields [11]. The stationary properties of the model are given in terms of the algebraic relations of the matrices appearing on the MPA. This ansatz was used in a variety of problems including interface growth [12], boundary induced phase transitions [10], [13]- [15], the dynamics of shocks [16] or traffic flow [17].

An important development of the MPA that appeared in the context of stochastic models is the dynamical matrix product ansatz (DMPA) $[18,30]$. This ansatz allows, whenever it is valid, the calculation of the probability densities, of the stochastic system, at arbitrary times. In the related spin Hamiltonian this DMPA asserts that not only the ground-state wave function, as in the standard MPA, but an arbitrary wavefunction have components expressed in terms of a matrix product ansatz whose matrices, in distinction of the standard MPA, are now time dependent. This DMPA was shown originally [18], [19] to be valid for the problem of asymmetric diffusion of particles on the lattice. More recently [20] it was also shown that this DMPA can also be formulated in the context of stochastic models with two species of hard-core particles. The condition of validity of the DMPA reproduces the subspace of parameters where the model is known to be exactly integrable. This fact induce us to conjecture that all the Hamiltonians, related or not to stochastic models, that are solvable through the Bethe ansatz may also be solvable by an appropriate MPA. This would mean that the components of the eigenfunctions of the exact integrable models, that according to the Bethe ansatz, are normally given by a combination of plane waves, can also be obtained from the algebraic properties of the matrices defining the MPA.

In this Letter we are going to show how the eigenspectra of the exact integrable quantum chains, with global conservation laws, can be obtained by an appropriate matrix-product ansatz. In this way we were able to obtain the integrability of several well known exact integrable models. Among the models with one global conservation law we have the XXZ chain [21], the spin$S$ Fateev-Zamolodchikov model [22], the Izergin-Korepin model [23], the solvable spin-1 model of Ref. [24], etc, and among the models with two global conservations we have the supersymmetric $t-J$ model [25], the spin-1 Sutherland and Perk-Schultz models $[26,27]$, the Hubbard model [28], as well the two-parameter integrable model presented in [29]. Moreover the matrix-product ansatz we propose enable us to show, with little effort, how to extend the above mentioned models, by including arbitrary range of hard-core interactions, without losing their exact integrability.

For brevity and in order to illustrate the proposed MPA we are going to present here two examples. The solution of the XXZ chain with arbitrary hard-core interactions among the up spins, as an example of a model with one global conservation law, and the solution of the Hubbard model as an example of a model with two global 
conservations laws.

The Hamiltonian of the XXZ chain with a hard-core exclusion of $S$ sites $(S=1,2, \ldots)$ is the anisotropic Heisenberg chain (anisotropy $\Delta$ ) where any two up spins are not allowed to occupy lattice sites at distances smaller than $S$. This Hamiltonian in a $L$-sites periodic chain is given by

$$
H_{S}=-\mathcal{P}_{S} \sum_{i=1}^{L}\left(\sigma_{i}^{x} \sigma_{i+1}^{x}+\sigma_{i}^{y} \sigma_{i+1}^{y}+\Delta \sigma_{i}^{z} \sigma_{i+1}^{z}\right) \mathcal{P}_{\mathcal{S}},
$$

where $\sigma^{x}, \sigma^{y}, \sigma^{z}$ are spin- $\frac{1}{2}$ Pauli matrices and $\mathcal{P}_{S}$ is a projector that projects out the configurations where any two up spins are at distances smaller than $S$. The case $S=1$ corresponds to the standard exactly solvable XXZ chain [21]. The conserved charge associated to the global conservation law of (1) is the $z$-component of the total magnetization, or equivalently the number of up spins (particles). The translation invariance of the lattice also ensures that the momentum is also a good quantum number.

The ansatz we propose states that any of the wavefunctions $\left|\psi_{n, P}\right\rangle$ in the sector with $n$ spins up $(n=$ $0,1,2, \ldots)$ and momentum $P=\frac{2 \pi l}{L}(l=0,1,2, \ldots, L-1)$ is given by a matrix product ansatz, i. e., their amplitudes are given by the trace of the matrix product:

$$
\begin{aligned}
& \mid \psi_{n, P}>=\sum_{x_{1}, \ldots, x_{n}}^{*} \operatorname{Tr}\left(E^{x_{1}-1} A E^{x_{2}-x_{1}-1} A \cdots\right. \\
& \left.\cdots E^{x_{n}-x_{n-1}-1} A E^{L-x_{n}} \Omega_{P}\right) \mid x_{1}, \ldots, x_{n}>,
\end{aligned}
$$

where $\left|x_{1}, \ldots, x_{n}\right\rangle$ denotes the configurations with up spins at $\left(x_{1}, \ldots, x_{n}\right)$ and the symbol $(*)$ in the sum means the restriction to the configurations where $\left|x_{i+1}-x_{i}\right| \geq S$. The algebraic properties of the matrices $A, E$ and $\Omega_{P}$ will be fixed by the eigenvalue equation

$$
H_{S}\left|\psi_{n, P}>=\varepsilon_{n}\right| \psi_{n, P}>
$$

The algebraic relations of the matrices $A$ and $E$ with $\Omega_{P}$ will fix the momentum of the eigenfunction. The fact that $\mid \psi_{n, P}>$ has a momentum $P$ imply that the ratio of the amplitudes corresponding to configurations $\mid x_{1}, \ldots, x_{n}>$ and $\left(\mid x_{1}+1, \ldots, x_{n}+1>\right.$ is $e^{-i P}$ and consequently from (2) we have the following relations

$$
A \Omega_{P}=e^{-i P} \Omega_{P} A, E \Omega_{P}=e^{-i P} \Omega_{P} A .
$$

The eigenvalue equation (3) when applied to the components of $\left|\psi_{n, P}\right\rangle$ where all the up spins are at distances larger than $S$ give us the constraint

$$
\begin{aligned}
& \varepsilon_{n} \operatorname{Tr}\left(\cdots E^{x_{i}-x_{i-1}-1} A E^{x_{i+1}-x_{i}-1} A \cdots A E^{L-x_{n}} \Omega_{P}\right) \\
& =-\sum_{i=1}^{n}\left[\operatorname{Tr}\left(\cdots E^{x_{i}-x_{i-1}-2} A E^{x_{i+1}-x_{i}} A \cdots A E^{L-x_{n}} \Omega_{P}\right)\right. \\
& \left.+\operatorname{Tr}\left(\cdots E^{x_{1}-x_{i-1}} A E^{x_{i+1}-x_{i}-2} A \cdots A E^{L-x_{n}} \Omega_{P}\right)\right] .
\end{aligned}
$$

A convenient solution of this last equation is obtained by identifying the matrices $A$ as composed by $n$ spectralparameter dependent matrices

$$
A=\sum_{j=1}^{n} A_{k_{j}} E^{2-S}
$$

where the matrices $A_{k_{j}}$ obey the commutation relations

$$
E A_{k_{j}}=e^{i k_{j}} A_{k_{j}} E, A_{k_{j}} \Omega_{P}=e^{-i P(S-1)} \Omega_{P} A_{k_{j}},
$$

and $k_{j}(j=1, \ldots, n)$ are in general complex numbers unknown a priori [31]. The energy and momentum are given respectively by

$$
\varepsilon_{n}=-\sum_{j=1}^{n}\left(e^{i k_{j}}+e^{-i k_{j}}\right), P=\sum_{i=1}^{n} k_{j} .
$$

The eigenvalue equation when applied to the other components of $\left|\psi_{n, P}\right\rangle$ will give the commutation relations of the matrices $\left\{A_{k_{j}}\right\}$ among themselves. In fact this algebra is obtained from the components where any pair of up spins are located at the closest positions ("matching conditions") $x_{j}$ and $x_{j+1}=x_{j}+S$, namely

$$
\begin{aligned}
& A_{k_{j}} A_{k_{l}}=S\left(k_{j}, k_{l}\right) A_{k_{j}} A_{k_{l}}(i \neq j), A_{k_{j}}^{2}=0, \\
& S\left(k_{j}, k_{l}\right)=-\frac{e^{i\left(k_{j}+k_{l}\right)}+1-2 \Delta e^{i k_{j}}}{e^{i\left(k_{j}+k_{l}\right)}+1-2 \Delta e^{i k_{l}}}, i, j=1, \ldots, n .
\end{aligned}
$$

No new algebraic relations appear for the matrices $A_{k_{j}}$ and the associativity of the algebra (7) and (9) follows from property $S\left(k_{i}, k_{j}\right) S\left(k_{j}, k_{i}\right)=1$. The cyclic property of the trace in (2), together with the algebraic relations (9) give us, for each $k_{j}(\mathrm{j}=1, \ldots, \mathrm{n})$, the conditions

$$
\begin{aligned}
& \operatorname{Tr}\left(A_{k_{1}} \cdots A_{k_{n}} E^{L-n(S-1)} \Omega_{P}\right)=e^{-i k_{j}(L-n S+n)} e^{-i P(S-1)} \\
& \times\left(\prod_{l=1}^{n} S\left(k_{j}, k_{l}\right)\right) \operatorname{Tr}\left(A_{k_{1}} \cdots A_{k_{n}} E^{L-n(S-1)} \Omega_{P}\right),
\end{aligned}
$$

that fix the up to now free complex spectral parameters $\left\{k_{j}\right\}$

$$
e^{i k_{j} L}=\prod_{l=1}^{n} S\left(k_{j}, k_{l}\right) e^{i\left(k_{j}-k_{l}\right)(S-1)},(j=1, \ldots, n) .
$$

The wavefunctions, obtained by using (2) (8) and (9) can be written as a combination of plane waves ("wave numbers" $\left\{k_{j}\right\}$ ), and apart from a normalization constant coincides with those obtained by the standard Bethe ansatz [21] for $S=1$ or arbitrary values of $S$ [32].

As a second example we consider the standard Hubbard model whose Hamiltonian in a periodic lattice with $L$ sites is given by

$$
H_{h}=-t \sum_{j, \sigma}\left(c_{j, \sigma}^{+} c_{j+1, \sigma}+c_{j+1, \sigma}^{+} c_{j, \sigma}\right)+u \sum_{j} \eta_{j,-} \eta_{j,+},
$$


where $c_{j, \sigma}^{+}$are creation operators of electrons of spin $\sigma= \pm$ at site $j$, and $\eta_{j, \sigma}=\sum_{j a} c_{j, \sigma}^{+} c_{j, \sigma}$ are the number operators of electrons of spin $\sigma$ at the site $j$. In this case we have two global conservation laws $n_{ \pm}$, corresponding to the number of electrons with spin $\sigma= \pm$, and the toal number of electrons is $n=n_{+}+n_{-}$. In order to form our MPA we associate, as before, the matrix $E$ to the empty sites, the matrices $X^{+}, X^{-}$to the single occupied sites with electrons with spin up and down, respectively, and the matrix $X^{0} \equiv X^{+} E^{-1} X^{-}$to the sites with double occupancy. Our MPA asserts that the components of the eigenfunction $\left|\psi_{n, P}\right\rangle$ of energy $\epsilon_{n}$ and momentum $P=2 \pi l / L(l=0,1, \ldots, L-1)$ corresponding to the configuration $\left|x_{1}, Q_{1} ; \ldots, x_{n}, Q_{n}\right\rangle$ where the nonempty sites $\left(x_{1}, \ldots, x_{n}\right)$ have occupation $\left(Q_{1}, \ldots, Q_{n}\right)$ $\left(Q_{i}=+,-, 0\right)$ will be given by the trace

$$
\operatorname{Tr}\left(E^{x_{1}-1} X^{Q_{1}} E^{x_{2}-x_{1}-1} X^{Q_{2}} \cdots X^{Q_{n}} E^{L-x_{n}} \Omega_{P}\right) .
$$

The momentum of the state $P$, as in (4), is fixed by imposing the commutation relations $X^{Q} \Omega_{P}=e^{-i P} \Omega_{P} X^{Q}$, $(Q=+,-, 0)$ and $E \Omega_{P}=e^{-i P} \Omega_{P} E$. The eigenvalue equation $H_{h}\left|\Psi_{n, P}>=\varepsilon_{n}\right| \Psi_{n, P}>$ will provide the algebraic relations of the matrices $E$ and $X^{Q}$.

The components of the wavefunction corresponding to the configurations where all the particles are at distances $\left|x_{i+1}-x_{i}\right|>1$ will give a generalization of (2) whose solution is obtained by introducing the convenient $n$ spectralparameter dependent matrices

$$
X^{Q}=\sum_{j=1}^{n} E X_{k_{j}}^{Q},(Q=+,-),
$$

whose commutation relations with the matrices $E$ and $\Omega_{P}$ are

$$
E X_{k_{j}}^{Q}=e^{i k_{j}} X_{k_{j}}^{Q} E, X_{k_{j}}^{Q} \Omega_{P}=\Omega_{P} X_{k_{j}}^{Q} .
$$

The energy and momentum in terms of these unknown complex spectral parameters are given, as in (8), by

$$
\varepsilon_{n}=-\sum_{j=1}^{n}\left(e^{i k_{j}}+e^{-i k_{j}}\right), \quad P=\sum_{j=1}^{n} k_{j} .
$$

The components where the particles occupy the closest positions and those where we have double occupancy, give us, by using (14) and (15) the algebraic relations

$$
\begin{aligned}
& X_{k_{l}}^{Q} X_{k_{j}}^{Q}=S_{Q Q}^{Q Q}\left(k_{l}, k_{j}\right) X_{k_{j}}^{Q} X_{k_{l}}^{Q}, \quad\left(X_{k_{j}}^{Q}\right)^{2}=0, \\
& X_{k_{l}}^{Q} X_{k_{j}}^{Q^{\prime}}=S_{Q Q^{\prime}}^{Q Q^{\prime}}\left(k_{l}, k_{j}\right) X_{k_{j}}^{Q^{\prime}} X_{k_{l}}^{Q}+S_{Q^{\prime} Q}^{Q Q^{\prime}}\left(k_{l}, k_{j}\right) X_{k_{j}}^{Q} X_{k_{l}}^{Q^{\prime}} \\
& S_{Q Q}^{Q Q}=-1, S_{Q^{\prime} Q}^{Q Q^{\prime}}\left(k_{l}, k_{j}\right)=-u e^{i\left(k_{l}+k_{j}\right)} / \alpha, \\
& S_{Q Q^{\prime}}^{Q^{\prime} Q}\left(k_{l}, k_{j}\right)=t\left(e^{i k_{l}}-e^{i k_{j}}\right)\left(1+e^{i\left(k_{l}+k_{j}\right)}\right) / \alpha, \\
& \alpha=u e^{i\left(k_{l}+k_{j}\right)}+t\left(1+e^{i\left(k_{l}+k_{j}\right)}\right)\left(e^{i k_{l}}-e^{i k_{j}}\right),
\end{aligned}
$$

where $Q^{\prime}=-Q$ and $Q= \pm$. The relations (14) and (16) define completely the algebra whose structural constants are the well know $S$-matrix of the Hubbard model [28]. Since the several components of the wavefunction should be uniquely related the above algebra should be associative. This associativity imply that the above $S$-matrix should satisfy the Yang-Baxter relations [33,2], which is indeed the case [28]. The components of the wavefunction corresponding to the configurations where we have three or four particles in next-neigboring sites would give in principle new relations involving three or four operators $X_{k_{j}}^{Q}$. These new relations are however consequences of the above relations (14) and (16). The cyclic property of the trace in (12) and the algebraic relations (14) and (16) will imply

$$
\begin{aligned}
& \operatorname{Tr}\left(X_{k_{1}}^{Q_{1}} \cdots X_{k_{j-1}}^{Q_{j-1}} X_{k_{j}}^{Q_{j}} \cdots X_{k_{n}}^{Q_{n}} E^{L} \Omega_{P}\right)=(-1)^{n} e^{i k_{j} L} \\
& \sum_{Q_{1}^{\prime}, \ldots, Q_{n}^{\prime}} \mathcal{T}\left(\{Q\},\left\{Q^{\prime}\right\}\right) \operatorname{Tr}\left(X_{k_{1}}^{Q_{1}^{\prime}} \cdots X_{k_{n}}^{Q_{n}^{\prime}} E^{L} \Omega_{P}\right) .
\end{aligned}
$$

where

$$
\mathcal{T}\left(\{Q\},\left\{Q^{\prime}\right\}\right)=\sum_{Q^{\prime \prime}, \ldots, Q{ }_{n}} \prod_{i=1}^{n} S_{Q_{i}^{\prime}, Q^{\prime}{ }_{i}{ }_{i}}^{Q_{i},{ }^{\prime}}
$$

is the transfer matrix of a non-homogeneous six-vertex model defined on a cylinder of perimeter $n$ and with Boltzman weights given by the $S$-matrices defined in (16). The eigenvalues of this auxiliary problem $\Lambda_{n}\left(k_{i} ; k_{1}, \ldots, k_{n}\right)$ can be obtained in a standard way by the coordinate-Bethe ansatz [21] or by the quantum inverse scattering method [34]. Using these eigenvalues in the relation (17) the spectral parameters $\left\{k_{j}\right\}$ will be fixed by the solutions of the system of equations

$$
e^{-i k_{j} L}=(-1)^{n} \Lambda_{n}\left(k_{j} ; k_{1}, \ldots, k_{n}\right),(j=1, \ldots, n) .
$$

These last equations coincide with the Bethe-Ansatz equations derived through the standard coordinate Bethe ansatz [28].

Generalizations of our MPA is quite simple to implement [35]. For example the solution of the excluded volume Hubbard model where electrons with spin up (down) exclude other electrons at $S_{+}\left(S_{-}\right)$sites on its right, but allows double occupancy at any site, is just obtained by changing in (13) $X^{Q}=\sum_{j} E^{S_{Q}+1} X_{k_{j}}^{Q}(Q= \pm)$.

We have also shown [35] that the above MPA also works for the other known exact integrable models with two conserved global quantities $(U(1) \otimes U(1))$, like the Essler-Korepin-Schoutens model [36] or the generalized two-parameter integrable model introduced in [29]. In those last cases the same MPA presented above for the Hubbard model apply except that now the matrices $X^{0}$ associated to the sites with double occupancy are given in terms of new operators $X^{0}=Y^{+} E^{-1} Y^{-}$, and we were able to rederive all the results obtained for those models through the standard Bethe ansatz. Hamiltonians with two global conservations that do not allow double 
occupancy like the stochastic Hamiltonian associated to the asymmetric diffusion with two kinds of particles [32], or the supersymmetric $t-J$ quantum chain [25] or the $S U(3)$ Sutherland and Perk-Schultz models [26,27], are obtained through the MPA (12), without the matrices $X^{0}$.

Models of spin one with a single global conservation law (conservation of the $z$-magnetization) like the FateevZamolodchikov model [22], the Izergin-Korepin model [23], or the solvable spin-1 Hamiltonian introduced in [29], have their solutions given by a MPA similar as in (2), where now we associate the matrices $E, A$ and $B E^{-1} B$ to the sites occupied by particles with spin $S^{z}=-1,0$ or +1 , respectively.

We have also obtained an appropriate extension of the presented MPA to non periodic, but exact integrable boundaries as, for example, the XXZ chain with surface fields.

It is interesting to notice that in the cases of exact integrable Hamiltonians associated to stochastic models, as in $[11,30]$, since we can write all eigenfunctions in a MPA, our results imply that we can equivalently write at any time the probability distribution of the model in terms of a time-dependent MPA, as happens in the DMPA [18].

In conclusion, we have shown that the eigenfunctions of a huge variety of exact integrable quantum chains can be expressed on an unified way in terms of a matrix product ansatz, whose matrices satisfy an associative algebra. The associativity of the algebra that warranties the exact integrability of the model is a consequence of the YangBaxter relations.

This work has been partially supported by FAPESP, CNPq and CAPES (Brazilian agencies).

[1] H. A. Bethe, Z. Phys. 71, 205 (1931).

[2] R. J. Baxter, Exactly solved models in statistical mechanics (Academic Press, New York, 1982).

[3] V. E. Korepin, A. G .Izergin and N. M. Bogoliubov, Quantum Inverse Scattering Method, Correlation Functions and Algebraic Bethe Ansatz (Cambridge University Press, Cambridge, 1992).

[4] F. H. L. Essler and V. E. Korepin, Exactly Solvable Models of Strongly Correlated Electrons (World Scientific, Singapore, 1994).

[5] P. Schlottmann, Int. J. Mod. Physics B, 11, 355 (1997).

[6] I. Affleck, T. Kennedy, E. H. Lieb and H. Tasaki, Commun. Math. Phys. 115, 477 (1988).

[7] D. P. Arovas, A. Auerbach, F. D. M. Haldane, Phys. Rev. Lett. 60, 531 (1998).

[8] M. Fannes, B. Nachtergaele, R. F. Werner, Commun. Math. Phys. 144, 443 (1992).

[9] A. Kluemper, A. Schadschneider and J. Zittarz, Z. Phys. B 87, 281 (1992); Europhys. Lett. 24, 293 (1993).
[10] B. Derrida, M. R. Evans, V. Hakim and V. Pasquier, J. Phys. A 26, 1493 (1993).

[11] F. C. Alcaraz, M. Droz, M. Henkel, and V. Rittenberg, Ann. Phys. (N.Y.) 230, 250 (1994).

[12] J. Krug and H. Spohn, Solids Far From Equilibrium Eds. C. Godrèche (cambridge University Press, Cambridge, 1991).

[13] B. Derrida, E. Domany and D. Mukamel, J. Stat. Phys. 69, 667 (1992).

[14] R. Domany and E. Schütz, J. Stat. Phys. 72, 277 (1993).

[15] M. R. Evans, D. P. Foster, C. Godrèche and D. Mukamel, J. Stat. Phys. 80, 69 (1995).

[16] B. Derrida, J. L. Lebowitz and E. R. Speer, J. Stat. Phys. 89, 135 (1997).

[17] K. Nagel and M. Schreckenberg, J. Physique 2, 2221 (1992)

[18] R. B. Stinchcombe and G. M. Schütz, Phys. Rev. Lett. 75, 140 (1995); Europhys. Lettt. 29, 663 (1995).

[19] T. Sasamoto and M. Wadati, J. Phys. Soc. Japan 66, 2618 (1999).

[20] V. Popkov, M. E. Fouladvand and G. M. Schütz, J. Phys. A 35, 7187 (2002).

[21] C. N. Yang and C. P. Yang, Phys. Rev. 150, 321 (1966).

[22] A. B. Zamolodchikov and V. Fateev, Sov. J. Nucl. Phys. 32, 298 (1980).

[23] A. G. Izergin and V. E. Korepin, Commun. Math. Phys. 79, 303 (1981).

[24] F. C. Alcaraz and R. Z. Bariev, J. Phys. A 34, L467 (2001).

[25] P. Schlottmann, Phys. Rev. B 36, 5177 (1987).

[26] B. Sutherland, Phys. Rev. B 12, 3795 (1975).

[27] J. H. H. Perk and C. L. Schultz, Phys. Lett. A 84, 407 (1981).

[28] E. H. Lieb and F. Y. Wu, Phys. Rev. Lett. 20, 1445 (1968).

[29] F. C. Alcaraz and R. Z. Bariev, J. Phys. A 32, L483 (1999).

[30] G. M. Schütz, in "Phase Transitions and Critical Phenomena", vol. 19, Edited by C. Domb and J. Lebowitz (Academic, London, 2000)

[31] Actually the most general relation $A=\sum_{j} E^{\alpha} A_{k_{j}} E^{\beta}$ with $(\alpha, \beta \in Z)$ could be used. However (6) is more convenient since the S-matrix in (9) will be independent from the hard-core size of the up spins.

[32] F. C. Alcaraz and R. Z. Bariev, in Statitical Physics in the Eve of the 21st Century, edited by M. T. Batchelor and L. T. Wille, Series on advances in Statistical Mechanics, Vol. 14 (World Scientic, Singapore, 1999), (Preprint cond-mat/9904042).

[33] C. N. Yang, Phys. Rev. Lett. 19, 1312 (1968).

[34] P.P. Kulish and N. Yu. Reshetikhin, Sov. Phys. -JETP 53, 108 (1981).

[35] F. C. Alcaraz and M. J. Lazo, to be published.

[36] F. H. L. Essler, V. E. Korepin and K. Schoutens, Phys. Rev. Lett. 68, 2960 (1992); Phys. Rev. Lett. 70, 73 (1993).

[37] F. C. Alcaraz and R. Z. Bariev, Braz. J. Phys. 30, 13 (2000). 